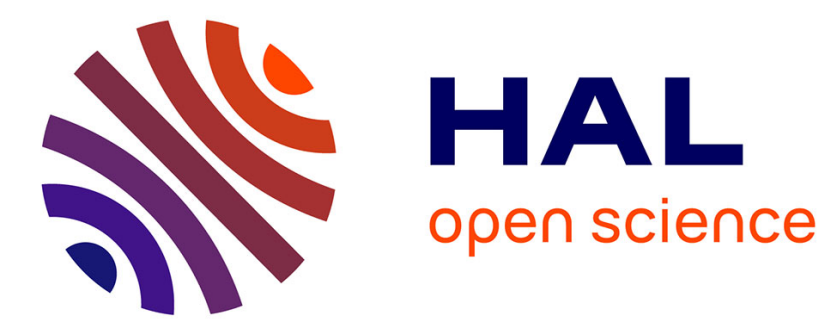

\title{
Positive Lower Bound for the Numerical Solution of a Convection-Diffusion Equation
}

\author{
Claire Chainais-Hillairet, Benoît Merlet, Alexis Vasseur
}

\section{To cite this version:}

Claire Chainais-Hillairet, Benoit Merlet, Alexis Vasseur. Positive Lower Bound for the Numerical Solution of a Convection-Diffusion Equation. FVCA8 2017 - International Conference on Finite Volumes for Complex Applications VIII, Jun 2017, Lille, France. pp.331-339, 10.1007/978-3-319-57397-7_26 . hal-01596076

\section{HAL Id: hal-01596076 https://hal.science/hal-01596076}

Submitted on 27 Sep 2017

HAL is a multi-disciplinary open access archive for the deposit and dissemination of scientific research documents, whether they are published or not. The documents may come from teaching and research institutions in France or abroad, or from public or private research centers.
L'archive ouverte pluridisciplinaire HAL, est destinée au dépôt et à la diffusion de documents scientifiques de niveau recherche, publiés ou non, émanant des établissements d'enseignement et de recherche français ou étrangers, des laboratoires publics ou privés. 


\title{
Positive lower bound for the numerical solution of a convection-diffusion equation
}

\author{
Claire Chainais-Hillairet, Benoît Merlet and Alexis F. Vasseur
}

\begin{abstract}
In this work, we apply a method due to De Giorgi [3] in order to establish a positive lower bound for the numerical solution of a stationary convection-diffusion equation.
\end{abstract}

Key words: finite volume scheme, isoperimetric inequality

MSC (2010): 65M08, 35B40.

\section{Introduction}

\section{The continuous problem}

Let $\Omega$ be an open bounded polygonal domain of $\mathbb{R}^{2}$. Let $f \in L^{\infty}(\Omega)$, we consider the following system of equations :

$$
\begin{aligned}
& -\Delta \Psi=f \quad \text { in } \Omega, \\
& \operatorname{div}(-\nabla v+\nabla \Psi v)=0 \quad \text { in } \Omega .
\end{aligned}
$$

This system is supplemented with mixed Dirichlet-Neumann boundary conditions. We assume that $\partial \Omega=\Gamma^{D} \cup \Gamma^{N}$ with $\Gamma^{D} \cap \Gamma^{N}=\emptyset$ and $\mathrm{m}\left(\Gamma^{D}\right)>0$ and we consider $v^{D} \in L^{\infty}\left(\Gamma^{D}\right), \Psi^{D} \in L^{\infty}\left(\Gamma^{D}\right)$. The boundary conditions write:

$$
\Psi=\Psi^{D}, v=v^{D} \text { on } \Gamma^{D} \text { and } \nabla \Psi \cdot \mathbf{n}=0=\nabla v \cdot \mathbf{n} \text { on } \Gamma^{N}
$$

Claire Chainais-Hillairet and Benoît Merlet

Univ. Lille, CNRS,UMR 8524-Laboratoire Paul Painlevé. F-59000 Lille, France

e-mail: Claire.Chainais@math.univ-lille1.fr, Benoit.Merlet@math.univ-lille1.fr

Alexis F. Vasseur

Department of Mathematics, University of Texas at Austin, C1200 Austin, TX 78712-0257

e-mail: vasseur@math.utexas.edu 
and $v^{D}$ is bounded from below by a positive constant. Up to a rescaling, we assume:

$$
v^{D} \geq 1 \text { a.e. on } \Gamma^{D} \text {. }
$$

With this hypothesis, one can prove, using De Giorgi's method (see $[3,5])$, that the weak solution $v$ to (1), (2) has a positive lower bound:

$$
\exists \alpha>0 \text { such that } v \geq \alpha \text { in } \Omega .
$$

Our aim is to adapt De Giorgi's method in a discrete setting. We establish that the approximation $v \mathscr{T}$ of $v$ obtained by one of the finite volume discretizations described below satisfies (4) with some $\alpha>0$ only depending on the continuous data and on the regularity of the mesh. De Giorgi's method is valid in any dimension and also for some parabolic systems. Here, for simplicity, we only treat the static case in dimension 2. The generalisation to evolutive convection-diffusion equations and drift-diffusion systems is the object of an ongoing work.

\section{The numerical scheme}

The mesh of the domain $\Omega, \mathscr{M}=(\mathscr{T}, \mathscr{E}, \mathscr{P})$, is classically given by: $\mathscr{T}$, a set of open polygonal control volumes, $\mathscr{E}$, a set of edges, $\mathscr{P}=\left(x_{K}\right)_{K \in \mathscr{T}}$ a set of points. As we deal with a Two-Point Flux Approximation (TPFA) of convection-diffusion equations, we assume that the mesh is admissible in the sense of [4] (Definition 9.1).

We distinguish in $\mathscr{E}$ the interior edges, $\sigma=K \mid L$, from the exterior edges: $\mathscr{E}=$ $\mathscr{E}_{\text {int }} \cup \mathscr{E}_{\text {ext }}$. Among the exterior edges, we distinguish the edges included in $\Gamma^{D}$ from the edges included in $\Gamma^{N}: \mathscr{E}_{e x t}=\mathscr{E}_{e x t}^{D} \cup \mathscr{E}_{e x t}^{N}$. For a given control volume $K \in \mathscr{T}$, we define $\mathscr{E}_{K}$ the set of its edges, which is also split into $\mathscr{E}_{K}=\mathscr{E}_{K, \text { int }} \cup \mathscr{E}_{K, \text { ext }} \cup \mathscr{E}_{K, \text { ext }}$. For each edge $\sigma \in \mathscr{E}$, we pick one cell in the non empty set $\left\{K: \sigma \in \mathscr{E}_{K}\right\}$ and denote it by $K_{\sigma}$. In the case of an interior edge $\sigma=K \mid L, K_{\sigma}$ is either $K$ or $L$.

For all edges $\sigma \in \mathscr{E}$, we set $\mathrm{d}_{\sigma}=\mathrm{d}\left(x_{K}, x_{L}\right)$ if $\sigma=K \mid L \in \mathscr{E}_{\text {int }}$ and $\mathrm{d}_{\sigma}=\mathrm{d}\left(x_{K}, \sigma\right)$ if $\sigma \in \mathscr{E}_{\text {ext }}$ with $\sigma \in \mathscr{E}_{K}$ and the transmissibility coefficient is defined by $\tau_{\sigma}=$ $\mathrm{m}(\sigma) / \mathrm{d}_{\sigma}$, for all $\sigma \in \mathscr{E}$. We assume that the mesh satisfies the regularity constraint:

$$
\exists \xi>0 \text { such that } \mathrm{d}\left(x_{K}, \sigma\right) \geq \xi \mathrm{d}_{\sigma}, \quad \forall K \in \mathscr{T}, \forall \sigma \in \mathscr{E}_{K} .
$$

The size of the mesh is defined by $h=\max \{\operatorname{diam}(K): K \in \mathscr{T}\}$.

Per se, a TPFA for a conservation law with unknown $u$ provides a vector $u_{\mathscr{T}}=\left(u_{K}\right)_{K \in \mathscr{T}}$ of approximate values and the associated piecewise constant function. However, since there are Dirichlet boundary conditions on a part of the boundary, we need to define approximate values for $u$ at the corresponding boundary edges: $u_{\mathscr{E} D}=\left(u_{\sigma}\right)_{\sigma \in \mathscr{E}_{e x t} D}$. Then, we set $u_{\mathscr{M}}=\left(u_{\mathscr{T}}, u_{\mathscr{E}} D\right)$ and we define

$$
D u_{K, \sigma}=u_{K, \sigma}-u_{K} \quad \text { and } \quad D_{\sigma} u=\left|D u_{K, \sigma}\right|, \quad \forall K \in \mathscr{T}, \forall \sigma \in \mathscr{E}_{K},
$$

where $u_{K, \sigma}$ is either $u_{L}(\sigma=K \mid L), u_{\sigma}\left(\sigma \in \mathscr{E}_{K, \text { ext }}\right)$ or $u_{K}\left(\sigma \in \mathscr{E}_{K, \text { ext }}\right)$.

For all $K \in \mathscr{T}, f_{K}$ denotes the mean value of $f$ over $K$. For all $\sigma \in \mathscr{E}_{\text {ext }}^{D}, \Psi_{\sigma}^{D}$ and $v_{\sigma}^{D}$ are respectively the mean values of $\Psi^{D}$ and $v^{D}$ over $\sigma$. We set: 


$$
v_{\sigma}=v_{\sigma}^{D}, \quad \Psi_{\sigma}=\Psi_{\sigma}^{D}, \quad \forall \sigma \in \mathscr{E}_{e x t}^{D} .
$$

We are now in the position to define the finite volume scheme for (1):

$$
\begin{gathered}
-\sum_{\sigma \in \mathscr{E}_{K}} \tau_{\sigma} D \Psi_{K, \sigma}=\mathrm{m}(K) f_{K}, \quad \forall K \in \mathscr{T}, \\
\sum_{\sigma \in \mathscr{E}_{K}} \mathscr{F}_{K, \sigma}=0, \quad \forall K \in \mathscr{T},
\end{gathered}
$$

where the numerical convection-diffusion fluxes $\mathscr{F}_{K, \sigma}$ are given by

$$
\mathscr{F}_{K, \sigma}=\tau_{\sigma}\left(B\left(-D \Psi_{K, \sigma}\right) v_{K}-B\left(D \Psi_{K, \sigma}\right) v_{K, \sigma}\right), \quad \forall K \in \mathscr{T}, \forall \sigma \in \mathscr{E}_{K}
$$

and $B$ is a Lipschitz-continuous function on $\mathbb{R}$ satisfying

$$
B(0)=1, \quad B(s)>0 \quad \text { and } \quad B(s)-B(-s)=-s \quad \forall s \in \mathbb{R} .
$$

The upwind scheme corresponds to the case $B(s)=1+s^{-}$and the ScharfetterGummel scheme to the case $B(s)=s /\left(e^{s}-1\right)$. They both satisfy (10).

\section{Main result}

The scheme (7)-(8)-(9) can be written as two linear systems of equations on the unknowns $\Psi_{\mathscr{T}}$ and $\mathbf{v}_{\mathscr{T}}: \mathbb{A} \Psi_{\mathscr{T}}=\mathbf{B}_{\Psi}$ and $\mathbb{M} v_{\mathscr{T}}=\mathbf{B}_{v}$. It is well-known that $\mathbb{A}$ is a positive-definite symmetric matrix and that $\mathbb{M}$ is an $\mathrm{M}$-matrix. Therefore, existence and uniqueness of a solution to the scheme is ensured. Moreover, the non negativity of the boundary condition $v^{D}$ implies $\mathbf{B}_{v} \geq 0$ and, since $\mathbb{M}$ is an M-matrix, we get $v_{\mathscr{T}} \geq 0$. We establish a positive lower bound for $v_{\mathscr{T}}$.

Theorem 1. There exists $h_{0}>0$ and $\alpha>0$ only depending on $\Omega, f, \Psi^{D}, v^{D}, \xi$ and $B$ such that if $0<h<h_{0}$, then

$$
v_{\mathscr{T}} \geq \alpha \quad \text { in } \Omega \text {. }
$$

The proof of Theorem 1 follows the lines of the proof at the continuous level. We introduce a sequence $\left(w_{\mathscr{M}}^{j}\right)_{j \geq 0}$ defined recursively by: $w_{\mathscr{M}}^{0}=1 / 2-v_{\mathscr{M}}$ and $w_{\mathscr{M}}^{j+1}=2 w_{\mathscr{M}}^{j}-1 / 2$ for all $j \geq 0$. A direct computation shows:

$$
w_{\mathscr{M}}^{j}=2^{j} w_{\mathscr{M}}^{0}-\left(2^{j}-1\right) / 2=1 / 2-2^{j} v_{\mathscr{M}}, \quad \forall j \geq 0 .
$$

It is clear that $w_{\mathscr{T}}^{j} \leq 1 / 2$ and that $w_{\mathscr{E} D}^{j} \leq-2^{j}+1 / 2<0$ for all $j \geq 0$. Moreover, if $w_{\mathscr{T}}^{j_{0}} \leq 1 / 4$ for some $j_{0} \geq 0$, we can conclude that $w_{\mathscr{T}}^{0} \leq 1 / 2-2^{-j_{0}-2}$ and (11) is established with $\alpha=2^{-j_{0}-2}$.

The proof then splits into three steps. In Section 2, we establish some discrete $a$ priori $H^{1}$-estimates on $\left[w_{\mathscr{M}}^{j}\right]^{+}$for $j \geq 0$. Then, we prove that the desired estimate $\left[w_{\mathscr{T}}^{j_{0}}\right]^{+} \leq 1 / 4$ can be obtained under a smallness assumption on $\left\|w_{\mathscr{T}}^{j_{0}}\right\|_{L^{2}}$. We conclude in Section 4 by proving that this smallness assumption is verified for some $j_{0}$. 
This is a consequence of the discrete $H^{1}$-estimate and of an isoperimetric inequality established in Section 3.

\section{Estimates on the approximate solution}

\section{$L^{\infty}$-estimate on the potential $\Psi$}

As $f \in L^{\infty}(\Omega)$, the solution $\Psi_{\mathscr{T}}$ to the scheme (8a) with (6) and (7) satisfies $L^{\infty}$ estimates, see for instance Lemma 6 in [2] and the references therein. These $L^{\infty}$ estimates imply a uniform bound on the $D_{\sigma} \Psi$ and, as $B$ is Lipschitz-continuous,

$$
\exists \beta>0 \text { such that } B\left(D_{\sigma} \Psi\right) \geq \beta \quad \forall \sigma \in \mathscr{E} .
$$

Let us note that $\beta=1$ for the upwind scheme $\left(B(s)=1+s^{-}\right)$.

\section{Energy estimates}

Thanks to (12), we have $v_{K}=2^{-j-1}-2^{-j} w_{K}^{j}$ for all $K \in \mathscr{T}$ and $j \geq 0$. Using (10), the numerical fluxes $\mathscr{F}_{K, \sigma}$ defined by (9) rewrite

$$
\mathscr{F}_{K, \sigma}=2^{-j-1} \tau_{\sigma} D \Psi_{K, \sigma}-2^{-j} \mathscr{G}_{K, \sigma}^{j} \quad \forall j \geq 0,
$$

where

$$
\mathscr{G}_{K, \sigma}^{j}=\tau_{\sigma}\left(B\left(-D \Psi_{K, \sigma}\right) w_{K}^{j}-B\left(D \Psi_{K, \sigma}\right) w_{K, \sigma}^{j}\right), \forall K \in \mathscr{T}, \forall \sigma \in \mathscr{E}_{K}, \forall j \geq 0 .
$$

Therefore, the scheme (8) implies:

$$
\sum_{\sigma \in \mathscr{E}_{K}} \mathscr{G}_{K, \sigma}^{j}=\frac{1}{2} \sum_{\sigma \in \mathscr{E}_{K}} \tau_{\sigma} D \Psi_{K, \sigma}=-\frac{1}{2} \mathrm{~m}(K) f_{K}, \quad \forall K \in \mathscr{T}, \forall j \geq 0 .
$$

Lemma 1. There exists $C_{0}$ only depending on $\Omega, \xi, \Psi^{D}, f$ and $B$ such that for every $j \geq 0$,

$$
\sum_{\sigma \in \mathscr{E}} \tau_{\sigma}\left(D_{\sigma}\left[w^{j}\right]^{+}\right)^{2} \leq C_{0} .
$$

Proof. Let us fix $j \geq 0$ and let us drop the superscript $j$. We use that $w_{\mathscr{M}}$ solves (14)(15) with $w_{\mathscr{E} D}^{+}=0$ to prove that $w_{\mathscr{T}}^{+}$satisfies (16).

Let us multiply (15) by $w_{K}^{+}$and sum over $K \in \mathscr{T}$. Rearranging the sum as a sum over edges and taking into account that $w_{\sigma}^{+}=0$ for $\sigma \in \mathscr{E}_{e x t}$ we get

$$
\sum_{\sigma \in \mathscr{E} ; K=K_{\sigma}} \mathscr{G}_{K, \sigma} D\left(w^{+}\right)_{K, \sigma}=\frac{1}{2} \sum_{K \in \mathscr{T}} \mathrm{m}(K) f_{K} w_{K}^{+} .
$$

But, thanks to (10), the numerical fluxes defined by (14) can be rewritten either as 


$$
\begin{aligned}
\mathscr{G}_{K, \sigma} & =\tau_{\sigma}\left[D \Psi_{K, \sigma} w_{K}-B\left(D \Psi_{K, \sigma}\right) D w_{K, \sigma}\right] \\
\text { or as } \quad \mathscr{G}_{K, \sigma} & =\tau_{\sigma}\left[D \Psi_{K, \sigma} w_{K, \sigma}-B\left(-D \Psi_{K, \sigma}\right) D w_{K, \sigma}\right] .
\end{aligned}
$$

We use the first expression when $D \Psi_{K, \sigma} \geq 0$ and the second one when $D \Psi_{K, \sigma}<0$. Combined with the inequalities $x\left(y^{+}-x^{+}\right) \leq \frac{1}{2}\left[\left(y^{+}\right)^{2}-\left(x^{+}\right)^{2}\right]$ and $(x-y)\left(x^{+}-\right.$ $\left.y^{+}\right) \geq\left(x^{+}-y^{+}\right)^{2}$, we get

$$
\mathscr{G}_{K, \sigma} D\left(w^{+}\right)_{K, \sigma} \leq \frac{1}{2} \tau_{\sigma} D \Psi_{K, \sigma}\left(D\left(w^{+}\right)^{2}\right)_{K, \sigma}-\tau_{\sigma} B\left(D_{\sigma} \Psi\right)\left(D_{\sigma} w^{+}\right)^{2} .
$$

Therefore, we deduce from (17), (18) and (8a):

$$
\sum_{\sigma \in \mathscr{E}} \tau_{\sigma} B\left(D_{\sigma} \Psi\right)\left(D_{\sigma} w^{+}\right)^{2} \leq \frac{1}{2} \sum_{K \in \mathscr{T}} \mathrm{m}(K) f_{K}\left(w_{K}^{+}\right)^{2}-\frac{1}{2} \sum_{K \in \mathscr{T}} \mathrm{m}(K) f_{K} w_{K}^{+} .
$$

Finally, since $B\left(D_{\sigma} \Psi\right) \geq \beta, w_{\mathscr{T}}^{+} \leq \frac{1}{2}$ and $f \in L^{\infty}$, we get (16).

From $L^{2}$-bound to $L^{\infty}$-bound for $w_{\mathscr{T}}^{j,+}$

We establish the desired bound $w_{\mathscr{T}}^{j} \leq \frac{1}{4}$ under a smallness assumption on $\left\|w_{\mathscr{T}}^{j,+}\right\|_{L^{2}}$.

Lemma 2. There exists $\delta>0$ only depending on $\Omega, f, \Psi^{D}$ and $\xi$ such that

$$
\sum_{K \in \mathscr{T}} \mathrm{m}(K)\left(w_{K}^{j,+}\right)^{2} \leq \delta^{2} \quad \Longrightarrow \quad w_{\mathscr{T}}^{j,+} \leq \frac{1}{4} .
$$

Proof. As above, we drop the superscript $j$ in the proof. For all $i \geq 0$, we set

$$
C_{i}=1 / 4-2^{-i-2}, \quad z_{\mathscr{M}}^{i}=\left(w_{\mathscr{M}}-C_{i}\right)^{+}, \quad E_{i}=\int_{\Omega}\left(z_{\mathscr{T}}^{i}\right)^{2}=\sum_{K \in \mathscr{T}} \mathrm{m}(K)\left(\left[w_{K}-C_{i}\right]^{+}\right)^{2} .
$$

We note that $C_{i}-C_{i-1}=2^{-i-2}$ and that $\left\{z_{\mathscr{T}}^{i}>0\right\} \subset\left\{z_{\mathscr{T}}^{i-1}>0\right\}$. Moreover,

$$
\begin{aligned}
& z_{\mathscr{T}}^{i-1}=z_{\mathscr{T}}^{i}+2^{-i-2} \quad \text { on }\left\{z_{\mathscr{T}}^{i}>0\right\} \\
& \text { and } \quad \int_{\Omega}\left(z_{\mathscr{T}}^{i-1}\right)^{2} \geq \int_{\Omega}\left(z_{\mathscr{T}}^{i}\right)^{2}+2^{-2(i+2)} \mathrm{m}\left(\left\{z_{\mathscr{T}}^{i}>0\right\}\right) .
\end{aligned}
$$

It yields

$$
E_{i} \leq E_{i-1} \quad \text { and } \quad \mathrm{m}\left(\left\{z_{\mathscr{T}}^{i}>0\right\}\right) \leq 2^{2(i+2)} E_{i-1} .
$$

Moreover, thanks to Young's inequality, we get

$$
\int_{\Omega} z_{\mathscr{T}}^{i} \leq \frac{1}{2} E_{i}+\frac{1}{2} \mathrm{~m}\left(\left\{z_{\mathscr{T}}^{i}>0\right\}\right) \leq \frac{1}{2}\left(1+2^{2(i+2)}\right) E_{i-1} .
$$

Applying now Hölder's inequality with $q \in(1,+\infty)$ and $q^{\prime}=q /(q-1)$, we get

$$
E_{i} \leq\left(\int_{\Omega}\left|z_{\mathscr{T}}^{i}\right|^{2 q}\right)^{1 / q} \mathrm{~m}\left(\left\{z_{\mathscr{T}}^{i}>0\right\}\right)^{1 / q^{\prime}}
$$


Since $z_{\mathscr{E} D}^{i}=0$, we apply a discrete Poincaré-Sobolev inequality to bound the integral (see Theorem 4.3 in [1]) and using (20) it yields

$$
E_{i} \leq \frac{C}{\xi}\left(\sum_{\sigma \in \mathscr{E}} \tau_{\sigma}\left(D_{\sigma} z^{i}\right)^{2}\right)\left(2^{2(i+2)} E_{i-1}\right)^{1 / q^{\prime}}
$$

with $C$ depending on $\Omega$ and $q$.

Let us now remark that the numerical fluxes $\mathscr{G}_{K, \sigma}$ defined by (14) rewrite as

$$
\mathscr{G}_{K, \sigma}=\tau_{\sigma}\left(B\left(-D \Psi_{K, \sigma}\right)\left(w_{K}-C_{i}\right)-B\left(D \Psi_{K, \sigma}\right)\left(w_{K, \sigma}-C_{i}\right)\right)+\tau_{\sigma} D \Psi_{K, \sigma} C_{i} .
$$

Then, following the proof of Lemma 1, we obtain

$$
\begin{aligned}
\sum_{\sigma \in \mathscr{E}} \tau_{\sigma} B\left(D_{\sigma} \Psi\right)\left(D_{\sigma} z^{i}\right)^{2} & \leq \frac{1}{2} \sum_{K \in \mathscr{T}} \mathrm{m}(K) f_{K}\left(z_{K}^{i}\right)^{2}-\left(\frac{1}{2}-C_{i}\right) \sum_{K \in \mathscr{T}} \mathrm{m}(K) f_{K} z_{K}^{i}, \\
& \leq \frac{1}{2}\|f\|_{\infty} E_{i}+\frac{1}{2}\|f\|_{\infty} \int_{\Omega} z_{\mathscr{T}}^{i} .
\end{aligned}
$$

Combined with (13), (20) and (21), this yields

$$
\sum_{\sigma \in \mathscr{E}} \tau_{\sigma}\left(D_{\sigma} z^{i}\right)^{2} \leq \frac{\|f\|_{\infty}}{\beta}\left(1+2^{2 i+3}\right) E_{i-1} .
$$

From (22), we deduce

$$
E_{i} \leq \frac{C}{\xi} \frac{\|f\|_{\infty}}{\beta}\left(1+2^{2 i+3}\right) 2^{2(i+2) / q} E_{i-1}^{1+1 / q^{\prime}} .
$$

Thus, $\left(E_{i}\right)_{i \geq 0}$ satisfies $0 \leq E_{i} \leq \kappa \lambda^{i}\left(E_{i-1}\right)^{\gamma}$ for $i \geq 0$ with $\kappa, \lambda \geq 0$ and $\gamma>1$. As shown for instance in [5], there exists $\delta>0$ such that if $E_{0} \in\left[0, \delta^{2}\right]$, then the sequence $\left(E_{i}\right)_{i \geq 0}$ converges to 0 . This proves the lemma.

\section{A discrete counterpart of De Giorgi's isoperimetric inequality}

Lemma 3. Let $w_{\mathscr{M}}=\left(w_{\mathscr{T}}, w_{\mathscr{E} D}\right)$ satisfying $w_{\mathscr{E} D} \leq 0$. Let us define:

$$
\mathscr{A}=\left\{x \in \Omega ; w_{\mathscr{T}}(x) \geq 1 / 4\right\} \quad \text { and } \quad \mathscr{B}=\left\{x \in \Omega ; 0<w_{\mathscr{T}}(x)<1 / 4\right\} .
$$

We assume that there exist $\mu>0$ and $C_{0}>0$ such that:

$$
\mathrm{m}(\mathscr{A}) \geq \mu \quad \text { and } \quad \sum_{\sigma \in \mathscr{E}} \tau_{\sigma}\left(D_{\sigma} w^{+}\right)^{2} \leq C_{0} .
$$

There exist $h_{0}>0$ and $\varepsilon>0$ only depending on $\xi, \Omega, \Gamma^{D}, C_{0}$ and $\mu$ such that if $h \leq h_{0}$ then $\mathrm{m}(\mathscr{B}) \geq \varepsilon$. 
Proof. Let us first recall Lemma 4.1 in [1] (see also Theorem 5.11.1 in [6]). There exists $C$ depending only on $\Omega$ and $\Gamma^{D}$ such that, for all $u_{\mathscr{T}} \in X(\mathscr{T})$ :

$$
\left\|u_{\mathscr{T}}\right\|_{L^{N /(N-1)}(\Omega)} \leq C\left(\sum_{\sigma \in \mathscr{E}_{\text {int }}} \mathrm{m}(\sigma)\left|D_{\sigma} u\right|+\sum_{\sigma \in \mathscr{E}_{\text {ext }}^{D}} \mathrm{~m}(\sigma)\left|u_{K}\right|\right) .
$$

For all $\zeta \in\left(0, \frac{1}{4}\right)$, we define the set $E_{\zeta}=\left\{x \in \Omega ; w_{\mathscr{T}}(x)>\zeta\right\}$ which contains $\mathscr{A}$. The characteristic function of $E_{\zeta}$ belongs to $X(\mathscr{T})$ and therefore verifies (24). As $N=2$ and using (23), we get:

$$
\sqrt{\mu} \leq\left(\mathrm{m}\left(E_{\zeta}\right)\right)^{1 / 2} \leq C\left(\sum_{\substack{\sigma=K \mid L \in \mathscr{E}_{i n} ; \\ w_{K} \leq \zeta<w_{L}}} \mathrm{~m}(\sigma)+\sum_{\substack{\sigma \in \mathscr{E}_{\text {ext }} ; \\ w_{K}>\zeta}} \mathrm{m}(\sigma)\right) .
$$

Let us introduce $\mathscr{C}=\left\{x \in \Omega ; w_{\mathscr{T}}(x) \leq 0\right\}$. For a Dirichlet boundary edge $\sigma \subset$ $\Gamma^{D}$ with $\sigma \in \mathscr{E}_{K}$, we write that $\sigma \in \mathscr{E}_{\text {ext }, \mathscr{A}}$ if $K \subset \mathscr{A}, \sigma \in \mathscr{E}_{\text {ext }, \mathscr{B}}$ if $K \subset \mathscr{B}$ and $\sigma \in \mathscr{E}_{\text {ext }, \mathscr{C}}$ if $K \subset \mathscr{C}$. Integrating (25) over $\zeta \in\left(0, \frac{1}{4}\right)$, we get :

$$
\begin{aligned}
& \frac{1}{4} \sqrt{\mu} \leq C\left(\sum_{\substack{\sigma=K \mid L \\
K \subset \mathscr{B}, L \subset \mathscr{B} \cup \mathscr{C}}} \mathrm{m}(\sigma)\left|w_{K}^{+}-w_{L}^{+}\right|+\sum_{\substack{\sigma=K \mid L \\
K \subset \mathscr{B}, L \subset \mathscr{A}}} \mathrm{m}(\sigma)\left|w_{K}^{+}-\frac{1}{4}\right|\right. \\
& \left.+\sum_{\substack{\sigma=K \mid L \\
K \subset \mathscr{A}, L \subset \mathscr{C}}} \frac{1}{4} \mathrm{~m}(\sigma)+\sum_{\sigma \in \mathscr{E}_{\text {ext }}^{D} \mathscr{B}} \mathrm{m}(\sigma)\left|w_{K}^{+}\right|+\sum_{\sigma \in \mathscr{E}_{\text {ext }, \mathscr{A}}} \mathrm{m}(\sigma) \frac{1}{4}\right) \\
& \leq C\left(\sum_{\substack{\sigma=K \mid L \\
K \subset \mathscr{B}, L \subset \mathscr{A} \cup \mathscr{B} \cup \mathscr{C}}} \mathrm{m}(\sigma)\left|w_{K}^{+}-w_{L}^{+}\right|+\sum_{\sigma \in \mathscr{E} D \text { ext }, \mathscr{B}} \mathrm{m}(\sigma)\left|w_{K}^{+}\right|\right. \\
& \left.+\sum_{\substack{\sigma=K \mid L \\
K \subset \mathscr{A}, L \subset \mathscr{C}}} \mathrm{m}(\sigma)\left|w_{K}^{+}-w_{L}^{+}\right|+\sum_{\sigma \in \mathscr{E}_{\text {ext }, \mathscr{A}}^{D}} \mathrm{~m}(\sigma)\left|w_{K}^{+}\right|\right) .
\end{aligned}
$$

We apply now Cauchy-Schwarz' inequality to the right-hand side, which yields:

$$
\frac{\mu}{16} \leq C C_{0}\left(\sum_{\sigma \subset \overline{\mathscr{B}}} \mathrm{m}(\sigma) \mathrm{d}_{\sigma}+\sum_{\substack{\sigma=K \mid L \\ K \subset \mathscr{A}, L \subset \mathscr{C}}} \mathrm{m}(\sigma) \mathrm{d}_{\sigma}+\sum_{\sigma \in \mathscr{E}_{\text {ext, } \mathscr{A}}} \mathrm{m}(\sigma) \mathrm{d}_{\sigma}\right) .
$$

On the one hand, the hypothesis (5) ensures that $\sum_{\sigma \subset \overline{\mathscr{B}}} \mathrm{m}(\sigma) \mathrm{d}_{\sigma} \leq \mathrm{m}(\mathscr{B}) / \xi$. On the other hand, if $\sigma=K \mid L$ with $K \subset \mathscr{A}$ and $L \subset \mathscr{C}$ or if $\sigma \in \mathscr{E}$ ext, $\mathscr{A}$, we have $\left|D_{\sigma} w^{+}\right| \geq$ $1 / 4$ and

$$
\sum_{\substack{\sigma=K \mid L \\ K \subset \mathscr{A}, L \subset \mathscr{C}}} \mathrm{m}(\sigma) \mathrm{d}_{\sigma}+\sum_{\sigma \in \mathscr{E}_{e x t, \mathscr{A}}} \mathrm{m}(\sigma) \mathrm{d}_{\sigma} \leq \frac{16 C_{0}}{\xi^{2}} h^{2} .
$$

Hence, $\frac{\mu}{16} \leq C C_{0}\left(\frac{\mathrm{m}(\mathscr{B})}{\xi}+\frac{16 C_{0}}{\xi^{2}} h^{2}\right)$, so that $\mathrm{m}(\mathscr{B}) \geq \frac{\xi \mu}{16 C C_{0}}-\frac{16 C_{0}}{\xi} h^{2}$. 


\section{Proof of Theorem 1}

Let $\delta>0$, given by Lemma 2, and let us assume that we have some $j_{0} \geq 0$ such that

$$
\sum_{K \in \mathscr{T}} \mathrm{m}(K)\left(\left[w_{K}^{j+1}\right]^{+}\right)^{2} \geq \delta^{2}, \quad \text { for } 0 \leq j \leq j_{0}-1 .
$$

Since $w_{\mathscr{T}}^{j} \leq \frac{1}{2}$ for all $j \geq 0$, we have

$$
\sum_{K \in \mathscr{T}} \mathrm{m}(K)\left(\left[w_{K}^{j+1}\right]^{+}\right)^{2} \leq \frac{1}{4} \mathrm{~m}\left(\left\{w_{\mathscr{T}}^{j+1} \geq 0\right\}\right)=\frac{1}{4} \mathrm{~m}\left(\left\{w_{\mathscr{T}}^{j} \geq \frac{1}{4}\right\}\right) .
$$

Hence, $\mathrm{m}\left(\left\{w_{\mathscr{T}}^{j} \geq 1 / 4\right\}\right) \geq 4 \delta^{2}$ for $0 \leq j \leq j_{0}$. Applying Lemma 3 with $\mu=4 \delta^{2}$ and $C_{0}$ given by Lemma 1 , there exists $\varepsilon=\varepsilon\left(C_{0}, \delta\right)>0$ such that if $h<h_{0}=h_{0}\left(C_{0}, \delta, \xi\right)$ we have $\mathrm{m}\left(\left\{0<w_{\mathscr{T}}^{j}<\frac{1}{4}\right\}\right) \geq \varepsilon$. Since $w_{\mathscr{T}}^{j+1} \leq 0$ if and only if $w_{\mathscr{T}}^{j} \leq \frac{1}{4}$, we deduce

$$
\mathrm{m}\left(\left\{w_{\mathscr{T}}^{j+1} \leq 0\right\}\right) \geq \mathrm{m}\left(\left\{w_{\mathscr{T}}^{j} \leq 0\right\}\right)+\mathrm{m}\left(\left\{0<w_{\mathscr{T}}^{j}<\frac{1}{4}\right\}\right) \geq \mathrm{m}\left(\left\{w_{\mathscr{T}}^{j} \leq 0\right\}\right)+\varepsilon .
$$

By induction, we get $\mathrm{m}\left(\left\{w_{\mathscr{T}}^{j_{0}} \leq 0\right\}\right) \geq \mathrm{m}\left(\left\{w_{\mathscr{T}}^{0} \leq 0\right\}\right)+j_{0} \varepsilon$ and since $\Omega$ is bounded,

$$
j_{0}<\mathrm{m}(\Omega) / \varepsilon
$$

We conclude that (26) is wrong for $j_{0}=j_{0}^{\star}:=\lceil\mathrm{m}(\Omega) / \varepsilon\rceil$. From Lemma 2, we get $w_{\mathscr{T}}^{j_{0}^{\star}} \leq 1 / 4$, that is $v_{\mathscr{T}} \geq 2^{-j_{0}^{\star}-2}$. This concludes the proof of Theorem 1 .

Acknowledgements: C. C.-H. and B. M. thank the team Inria/Rapsodi and the Labex CEMPI (ANR-11-LABX-0007-01) for their support. The authors thank the referees for their careful reading.

\section{References}

1. Bessemoulin-Chatard, M., Chainais-Hillairet, C., Filbet, F.: On discrete functional inequalities for some finite volume schemes. IMA J. Numer. Anal. (2014)

2. Chainais-Hillairet, C., Gisclon, M., Jüngel, A.: A finite-volume scheme for the multidimensional quantum drift-diffusion model for semiconductors. Numer. Methods Partial Differential Equations 27(6), 1483-1510 (2011)

3. De Giorgi, E.: Sulla differenziabilità e l'analiticità delle estremali degli integrali multipli regolari. Mem. Accad. Sci. Torino. Cl. Sci. Fis. Mat. Nat. (3) 3, 25-43 (1957)

4. Eymard, R., Gallouët, T., Herbin, R.: Finite volume methods. In: Handbook of numerical analysis, vol. VII, pp. 713-1020. North-Holland, Amsterdam (2000)

5. Vasseur, A.F.: The De Giorgi method for elliptic and parabolic equations and some applications. In: Lectures on the analysis of nonlinear partial differential equations. Part 4, Morningside Lect. Math., vol. 4, pp. 195-222. Int. Press, Somerville, MA (2016)

6. Ziemer, W.P.: Weakly differentiable functions, Graduate Texts in Mathematics, vol. 120. Springer-Verlag, New York (1989) 